\title{
Lightlike Hypersurfaces of an Indefinite Para Sasakian Manifold
}

\author{
Selcen YÜKSEL PERKTAŞ ${ }^{1, *}$, Erol KILIÇ ${ }^{2}$, Mukut Mani TRIPATHI ${ }^{3}$ \\ ${ }^{1}$ Adlyaman University, Faculty of Arts and Science, Department of Mathematics, Adlyaman, Turkey \\ sperktas@adiyaman.edu.tr,ORCID:0000-0002-8848-0621 \\ ${ }^{2}$ Inönü University, Faculty of Arts and Science, Department of Mathematics, Malatya, Turkey \\ erol.kilic@inonu.edu.tr,ORCID:0000-0001-7536-0404 \\ ${ }^{3}$ Banaras Hindu University, Institute of Science, Department of Mathematics, Varanasi, India \\ mmtripathi66@yahoo.com,ORCID:0000-0002-6113-039X
}

\begin{abstract}
In this paper, we initiate the study of lightlike hypersurfaces of an indefinite almost paracontact metric manifold which are tangent to the structure vector field. In particular, we give definitions of invariant lightlike hypersurfaces and screen semi-invariant lightlike hypersurfaces, and give some examples. Integrability conditions for the distributions involved in the screen semi-invariant lightlike hypersurface of an indefinite para Sasakian manifold are investigated.
\end{abstract}

Keywords: Indefinite almost paracontact manifold, Indefinite para Sasakian manifold, Invariant lightlike hypersurface, Screen semi-invariant lightlike hypersurface

\section{Bir Belirsiz Para Sasakiyan Manifoldun Lightlike Hiperyüzeyleri}

$\ddot{O} z$

Bu makalede, bir belirsiz para Sasakiyan manifoldun yapı vektör alanına teğet olan lightlike hiperyüzeyleri çalışılmıştır. Özel olarak, değişmez lightlike hiperyüzeyler ve ekran yarı-değişmez lightlike hiperyüzeyler tanıtılarak bazı örnekler verilmiştir. Bir belirsiz para Sasakian manifoldun ekran yarı-değișmez lightlike hiperyüzeyine dahil olan distribüsyonlar için integrallenebilirlik koşulları incelenmiştir.

\footnotetext{
* Corresponding Author
} 
Anahtar Kelimeler: Belirsiz hemen hemen parakontakt manifold, Belirsiz para Sasakiyan manifold, Değişmez lightlike hiperyüzey, Ekran yarı-değişmez lightlike hiperyüzey

\section{Introduction}

The geometry of submanifolds of semi-Riemannian manifolds has been one of the most active research area in differential geometry. A submanifold of a semi-Riemannian manifold is called a lightlike submanifold provided the induced metric on the submanifold is degenerate. In case of a lightlike submanifold, intersection of the normal vector bundle and the tangent vector bundle is non-trivial, and therefore its study evolves to be quite different from that of the nondegenerate submanifolds. However, the existence of a unique lightlike line subbundle of tangent vector bundle along every lightlike submanifold enables us partially to overcome the problems arising from such difficulty. In [1], the author obtained a canonical Riemannian vector bundle along the lightlike submanifold, which enables to study such submanifolds using the methods of Riemannian geometry, by dividing out the lightlike line subbundle from tangent vector bundle. For further read we refer $[2,3]$.

An almost paracontact structure $(\breve{\Psi}, \breve{\xi}, \breve{\eta})$, an analogue of the almost contact structure [4, 5], satisfying $\breve{\Psi}^{2}=I-\breve{\eta} \otimes \breve{\xi}$ and $\breve{\eta}(\breve{\xi})=1$ on a smooth manifold, was introduced by I. Sāto [6]. An almost contact structure is closely related to an almost complex structure and an almost contact manifold is always odd-dimensional, but an almost paracontact structure is closely related to an almost product structure and a manifold equipped with such an structure can be even-dimensional also. In 1969, T. Takahashi [7] proposed the idea of almost contact manifolds equipped with an associated pseudo-Riemannian metric (a.k.a. ( $(\varepsilon)$-almost contact metric manifolds) and studied Sasakian manifolds endowed with an associated pseudo-Riemannian metric (a.k.a. $(\varepsilon)$ Sasakian manifolds) (see [8-10]). The study of lightlike hypersurfaces of indefinite Sasakian manifolds were initiated in [11]. Later, in 2007 lightlike submanifolds of indefinite Sasakian manifolds were studied [10].

An indefinite almost contact metric manifold is always odd-dimensional. On the other hand, in a Lorentzian almost paracontact manifold proposed by K. Matsumoto [12], 
the compatible semi-Riemannian metric is of index 1 and the structure vector field is always timelike. Motivated by these circumstances, the authors of [13] associated a semiRiemannian metric, not necessarily Lorentzian, with an almost paracontact structure. The resulted structure is called an indefinite almost paracontact structure, where the structure vector field may be spacelike or timelike (see also [14]). The authors in [15] and [16] studied lightlike submanifolds of indefinite para-Sasakian manifolds admitting a spacelike characteristic vector field.

In the present paper, as a first step to study lightlike geometry of indefinite almost paracontact metric manifolds we study lightlike hypersurfaces. We organize the paper as follows. In section 2, we give a brief account of lightlike hypersurfaces of a semiRiemannian manifold, for later use. Section 3 is devoted to indefinite almost paracontact metric manifolds. Section 4 contains investigations of lightlike hypersurfaces of indefinite almost paracontact metric manifolds. In section 5, we define invariant lightlike hypersurfaces and give an example. Screen semi invariant hypersurfaces are introduced in Section 6. Moreover, integrability conditions for the distributions involved in the screen semi-invariant lightlike hypersurface are investigated when the ambient manifold is an indefinite para Sasakian manifold.

\section{Lightlike Hypersurfaces}

Suppose $N$ is a hypersurface in an $(n+2)$-dimensional semi-Riemannian manifold $(\breve{N}, \breve{g})$ of fixed index $q \in\{1, \ldots, n+1\}$ with the property that the induced metric $g=$ $\left.\breve{g}\right|_{N}$ on the hypersurface be degenerate. Then there exists a vector field $E \neq 0$ on $N$ satisfying

$$
g(E, X)=0, \quad X \in \Gamma(T N) \text {. }
$$

The radical space [17] of $T_{p} N$, at each point $p \in N$, is defined by

$$
\operatorname{Rad}_{p} N=\left\{E \in T_{p} N: g(E, X)=0, \quad X \in \Gamma\left(T_{p} N\right)\right\},
$$

and its dimension is called the nullity degree of $g$. In this case $(N, g)$ is called a lightlike hypersurface of $(\breve{N}, \breve{g})$ and

$$
\operatorname{RadT}_{p} N=T_{p} N \cap T_{p} N^{\perp}
$$


Since $\operatorname{dim} T_{x} N^{\perp}=1$, then we have

$$
\operatorname{dim} \operatorname{Rad}_{p} N=1, \quad \operatorname{RadT}_{p} N=T_{p} N^{\perp}
$$

The distribution RadTN is known as the radical distribution, which is spanned by the null vector field $E$.

We decompose $T N$ into orthogonal direct sum

$$
T N=S(T N) \perp \operatorname{RadTN}
$$

where $S(T N)$ a complementary vector bundle of RadTN in $T N$, known as the screen distribution on $N$. Because of non-degeneracy of $S(T N)$, there exists the screen transversal bundle $S(T N)^{\perp}$ of rank 2 , which is a complementary orthogonal vector subbundle to $S(T N)$ in $T \breve{N}$.

Since $\operatorname{RadTN}$ is a lightlike vector subbundle of $S(T N)^{\perp}$, therefore for any local section $E \in \Gamma(\operatorname{RadTN})$ we have a unique local section $\zeta$ of $S(T N)^{\perp}$ such that

$$
\breve{g}(\zeta, \zeta)=0, \quad \breve{g}(E, \zeta)=1 .
$$

Hence, $\zeta$ is not tangent to $N$ and $\{E, \zeta\}$ is a local frame field of $S(T N)^{\perp}$. Moreover, we obtain a 1-dimensional vector subbundle $\operatorname{ltr} T N$ of $T \breve{N}$, namely lightlike transversal bundle, which is locally spanned by $\zeta$. Then we set

$$
S(T N)^{\perp}=\operatorname{RadTN} \oplus \operatorname{ltr} T N
$$

where the decomposition is not orthogonal. Thus we have the following decomposition of $T \breve{N}$ :

$$
T \breve{N}=S(T N) \perp \operatorname{RadTN} \oplus \operatorname{ltr} T N=T N \oplus \operatorname{ltr} T N
$$

The decomposition (5) of $\breve{N}$ along a lightlike hypersurface $N$ entails the following Gauss and Weingarten formulas, respectively:

$$
\begin{aligned}
& \breve{\nabla}_{X} Y=\nabla_{X} Y+B(X, Y) \zeta, \\
& \breve{\nabla}_{X} \zeta=-A_{\zeta} X+v(X) \zeta,
\end{aligned}
$$


where $B$ is a second order symmetric tensor field called the second fundamental form, $A$ is an endomorphism of $T N$ called the shape operator with respect to $\zeta$ and $v$ is a 1 -form on $N$ [2]. For each $X \in \Gamma(T N)$, we may write

$$
X=f X+w(X) E,
$$

where $f$ is the projection of $T N$ on $S(T N)$ and $w$ a 1 -form given by

$$
w(X)=\breve{g}(X, \zeta)
$$

From (7), for all $X, Y, Z \in \Gamma(T N)$, we get

$$
\left(\nabla_{X} g\right)(Y, Z)=B(X, Y) w(Z)+B(X, Z) w(Y)
$$

The aforementioned relation shows that the induced connection $\nabla$ on $N$ is non-metric. From (3), we have

$$
\begin{gathered}
\nabla_{X} W=\nabla_{X}^{*} W+C(X, W) E, \\
\nabla_{X} E=-A_{E}^{*} X-v(X) E,
\end{gathered}
$$

for all $X \in \Gamma(T N), W \in \Gamma(S(T N))$, where $C, A_{E}^{*}$ and $\nabla^{*}$ denote the local second fundamental form, the local shape operator and the induced connection on $S(T N)$, respectively. Note that $\nabla_{X}^{*} W$ and $A_{E}^{*} X$ belong to $\Gamma(S(T N))$. Also, we have the following identities

$$
\begin{gathered}
g\left(A_{E}^{*} X, W\right)=B(X, W), \\
g\left(A_{E}^{*} X, \zeta\right)=0 \\
B(X, E)=0 \\
g\left(A_{\zeta} X, \zeta\right)=0 .
\end{gathered}
$$

In view of (13) and (15) one gets

$$
A_{E}^{*} E=0
$$

For details we refer to $[2,3]$ and [18]. 


\section{Indefinite Almost Paracontact Metric Manifolds}

Let $\widetilde{N}$ be an almost paracontact manifold [6] endowed with an almost paracontact structure $(\breve{\Psi}, \breve{\xi}, \breve{\eta})$ consisting of a tensor field $\breve{\Psi}$ of type $(1,1)$, a vector field $\breve{\xi}$ and a 1 form $\breve{\eta}$ satisfying

$$
\begin{gathered}
\breve{\Psi}^{2}=I-\breve{\eta} \otimes \breve{\xi}, \\
\breve{\eta}(\breve{\xi})=1, \\
\breve{\Psi} \breve{\xi}=0, \\
\breve{\eta} \circ \breve{\Psi}=0 .
\end{gathered}
$$

It is easy to verify that (18) and one of (19), (20) and (21) imply the remaining two equations. Let $\breve{g}$ be a semi-Riemannian metric [17] such that

$$
\breve{g}(\breve{\Psi} X, \breve{\Psi} Y)=\breve{g}(X, Y)-\varepsilon \breve{\eta}(X) \breve{\eta}(Y), \quad X, Y \in \Gamma(T \breve{N}),
$$

where $\varepsilon= \pm 1$. Then $\widetilde{N}$ is called an indefinite almost paracontact metric manifold equipped with an indefinite almost paracontact metric structure $(\breve{\Psi}, \breve{\xi}, \breve{\eta}, \breve{g}, \varepsilon)$ [13]. In particular, if the metric is a Lorentzian metric [19], then an indefinite almost paracontact metric manifold is called a Lorentzian almost paracontact manifold. From (22) we have

$$
\breve{g}(X, \breve{\Psi} Y)=\breve{g}(\breve{\Psi} X, Y)
$$

along with

$$
\breve{g}(X, \breve{\xi})=\varepsilon \breve{\eta}(X)
$$

for all $X, Y \in \Gamma(T \breve{N})$. From (24) it follows that

$$
\breve{g}(\breve{\xi}, \breve{\xi})=\varepsilon,
$$

that is, the structure vector field $\breve{\xi}$ cannot be lightlike.

Let $(\breve{N}, \breve{\Psi}, \breve{\xi}, \breve{\eta}, \breve{g}, \varepsilon)$ be an indefinite almost paracontact metric manifold (resp. a Lorentzian almost paracontact manifold). If $\varepsilon=1$, then $\widetilde{N}$ will be said to be a spacelike indefinite almost paracontact metric manifold (resp. a spacelike Lorentzian almost 
paracontact manifold). Similarly, if $\varepsilon=-1$, then $\widetilde{N}$ will be said to be a timelike indefinite almost paracontact metric manifold (resp. a timelike Lorentzian almost paracontact manifold) [13].

An indefinite almost contact metric structure is called an indefinite para Sasakian structure if

$$
\left(\breve{\nabla}_{X} \breve{\Psi}\right) Y=-\breve{g}(\breve{\Psi} X, \breve{\Psi} Y) \breve{\xi}-\varepsilon \breve{\eta}(Y) \breve{\Psi}^{2} X, \quad X, Y \in \Gamma(T \breve{N})
$$

where $\breve{\nabla}$ is the Levi-Civita connection with respect to $\breve{g}$. A manifold endowed with an indefinite para Sasakian structure is called an indefinite para Sasakian manifold [13]. In an indefinite para Sasakian manifold, we have

$$
\begin{gathered}
\breve{\nabla} \breve{\xi}=\varepsilon \breve{\Psi}, \\
\breve{\Phi}(X, Y)=\breve{g}(\breve{\Psi} X, Y)=\varepsilon \breve{g}\left(\breve{\nabla}_{X} \breve{\xi}, Y\right)=\left(\breve{\nabla}_{X} \breve{\eta}\right) Y, \quad X, Y \in \Gamma(T \breve{N}),
\end{gathered}
$$

where

$$
\breve{\Phi}(X, Y)=\breve{g}(X, \breve{\Psi} Y)
$$

From (29) we have

$$
\begin{aligned}
& \breve{\Phi}(X, \breve{\xi})=0, \\
& \breve{\Phi}(\breve{\Psi} X, \breve{\Psi} Y)=\breve{\Phi}(X, Y), \\
& \breve{\Phi}(X, \breve{\Psi} Y)=\breve{\Phi}(\breve{\Psi} X, Y)
\end{aligned}
$$

for all $X, Y \in \Gamma(T \breve{N})$.

In an indefinite para Sasakian manifold $\breve{N}$, Riemannian curvature tensor $\breve{R}$ and Ricci tensor $\breve{S}$ of $\breve{N}$ satisfy [13]

$$
\begin{gathered}
\breve{R}(X, Y) \breve{\xi}=\breve{\eta}(X) Y-\breve{\eta}(Y) X, \\
\breve{R}(X, Y, Z, \breve{\xi})=-\breve{\eta}(X) \breve{g}(Y, Z)+\breve{\eta}(Y) \breve{g}(X, Z), \\
\breve{\eta}(\breve{R}(X, Y) Z)=-\varepsilon \breve{\eta}(X) \breve{g}(Y, Z)+\varepsilon \breve{\eta}(Y) \breve{g}(X, Z),
\end{gathered}
$$




$$
\begin{gathered}
\breve{R}(\breve{\xi}, X) Y=-\varepsilon \breve{g}(X, Y) \breve{\xi}+\breve{\eta}(Y) X, \\
\breve{S}(Y, \breve{\xi})=-(n-1) \breve{\eta}(Y),
\end{gathered}
$$

for all $X, Y, Z \in \Gamma(T \widetilde{N})$.

\section{Lightlike Hypersurfaces of Indefinite Para Sasakian Manifolds}

Let $N$ be a lightlike hypersurface of an indefinite para Sasakian manifold $(\breve{N}, \breve{\Psi}, \breve{\xi}, \breve{\eta}, \breve{g}, \varepsilon)$ of dimension $(n+2)$, tangent to $\breve{\xi}$. In this case, the non-null vector field $\breve{\xi}$ must belong to the screen distribution $S(T N)$. Moreover, $\breve{\xi}$ must be spacelike, that is, $\breve{N}$ must be a spacelike para Sasakian manifold.

Let $E$ be a local section of RadTN and $\zeta$ a local section of ltrTN. In view of (24), we have

$$
\breve{\eta}(E)=0, \quad \breve{\eta}(\zeta)=0 .
$$

From (22), it is easy to see that $\breve{\Psi} E$ and $\breve{\Psi} \zeta$ are lightlike vector fields and

$$
\breve{\Psi}^{2} E=E, \quad \breve{\Psi}^{2} \zeta=\zeta
$$

Now, for $X \in \Gamma(T N)$, we write

$$
\breve{\Psi} X=\phi X+\mu(X) N
$$

where $\phi X \in \Gamma(T N)$ and

$$
\mu(X)=\breve{g}(\breve{\Psi} X, E)=\breve{g}(X, \breve{\Psi} E)
$$

Proposition 1. Let $N$ be a lightlike hypersurface of an $(n+2)$-dimensional indefinite almost paracontact metric manifold $(\breve{N}, \breve{\Psi}, \breve{\xi}, \breve{\eta}, \breve{g}, \varepsilon)$, tangent to $\breve{\xi}$. Then

$$
\begin{aligned}
& \breve{g}(\breve{\Psi} E, E)=0, \\
& \breve{g}(\breve{\Psi} E, \zeta)=\varepsilon g\left(A_{\zeta} E, \breve{\xi}\right) .
\end{aligned}
$$

Proof. The equations (27) and (17) imply (40). By using (27), (4) and (7) we obtain

$$
\varepsilon \breve{g}(\breve{\Psi} E, \zeta)=\breve{g}\left(\left(\breve{\nabla}_{E} \breve{\xi}, \zeta\right)=-\breve{g}\left(\breve{\zeta}, \breve{\nabla}_{E} \zeta\right)=g\left(A_{\zeta} E, \breve{\xi}\right),\right.
$$


which implies (41).

Remark 2. From (40) we see that there is no component of $\breve{\Psi} E$ in $\operatorname{ltr} T N$, thus $\breve{\Psi} E \in \Gamma(T N)$. Moreover, (41) implies that there may be a component of $\breve{\Psi} E$ in RadTN. Thus, in view of (8) and (40), we observe that

$$
\breve{\Psi} E=\phi E=f \breve{\Psi} E+w(\breve{\Psi} E) E .
$$

Proposition 3. Let $N$ be a lightlike hypersurface of an $(n+2)$-dimensional indefinite almost paracontact metric manifold $(\breve{N}, \breve{\Psi}, \breve{\xi}, \breve{\eta}, \breve{g}, \varepsilon)$, tangent to $\breve{\xi}$. Then

$$
\begin{gathered}
g(X, \phi Y)=g(\phi X, Y)+(\mu \wedge w)(X, Y) \\
g(X, Y)=g(\phi X, \phi Y)+\varepsilon \breve{\eta}(X) \breve{\eta}(Y)+\mu(X) w(\phi Y)+\mu(Y) w(\phi X),
\end{gathered}
$$

for all $X, Y \in \Gamma(T N)$.

Proof. From (38) and (39), we get

$$
\breve{g}(\breve{\Psi} X, Y)=g(\phi X, Y)+\mu(X) w(Y) .
$$

Hence in view of (23) we get (43). Using (38) we have

$$
\breve{g}(\breve{\Psi} X, \breve{\Psi} Y)=g(\phi X, \phi Y)+\mu(X) w(\phi Y)+\mu(Y) w(\phi X)
$$

Thus by using (45) and (22) we complete the proof.

Corollary 4. Suppose that $N$ is a lightlike hypersurface of an $(n+2)$-dimensional indefinite almost paracontact metric manifold $(\breve{N}, \breve{\Psi}, \breve{\xi}, \breve{\eta}, \breve{g}, \varepsilon)$, tangent to $\breve{\xi}$. Then

$$
g(\breve{\xi}, \phi X)=0, \quad X \in \Gamma(T N)
$$

Proposition 5. If $N$ is a lightlike hypersurface of an $(n+2)$-dimensional indefinite para Sasakian manifold $(\breve{N}, \breve{\Psi}, \breve{\xi}, \breve{\eta}, \breve{g}, \varepsilon)$, which is tangnet to $\breve{\xi}$, then

$$
\begin{gathered}
\phi^{2} X=X-\breve{\eta}(X) \breve{\xi}-\mu(\phi X) \zeta-\mu(X) \breve{\Psi} \zeta, \\
\phi X=\varepsilon \nabla_{X} \breve{\xi}, \\
B(X, \breve{\xi})=\varepsilon \mu(X),
\end{gathered}
$$


for any $X \in \Gamma(T N)$.

Proof. From (38) and (18), we get (46). Next, from (27), (6) and (38) we obtain

$$
\varepsilon \nabla_{X} \breve{\xi}+\varepsilon B(X, \breve{\xi}) \zeta=\phi X+\mu(X) \zeta
$$

Now, equating tangential parts in (49), one gets (47). Similarly, equating transversal parts in (49), one gets (48).

\section{Invariant Lightlike Hypersurfaces}

We begin with the following.

Definition 6. A lightlike hypersurface $N$ of an $(n+2)$-dimensional indefinite almost paracontact metric manifold $(\breve{N}, \breve{\Psi}, \breve{\xi}, \breve{\eta}, \breve{g}, \varepsilon)$ is said to be invariant if $\breve{\Psi}(S(T N))=S(T N)$.

Example 7. Let $\mathrm{R}^{5}$ be the 5 -dimensional real number space with a coordinate system $(x, y, z, t, s)$. We define

$$
\begin{gathered}
\breve{\eta}=d s-y d x-t d z, \quad \breve{\xi}=\frac{\partial}{\partial s} \\
\breve{\Psi}\left(\frac{\partial}{\partial x}\right)=-\frac{\partial}{\partial x}-y \frac{\partial}{\partial s}, \quad \breve{\Psi}\left(\frac{\partial}{\partial y}\right)=-\frac{\partial}{\partial y}, \\
\breve{\Psi}\left(\frac{\partial}{\partial z}\right)=-\frac{\partial}{\partial z}-t \frac{\partial}{\partial s}, \quad \breve{\Psi}\left(\frac{\partial}{\partial t}\right)=-\frac{\partial}{\partial t}, \quad \breve{\Psi}\left(\frac{\partial}{\partial s}\right)=0, \\
\breve{g}=-(d x)^{2}-(d y)^{2}+(d z)^{2}+(d t)^{2}+(d s)^{2} \\
-t(d z \otimes d s+d s \otimes d z)-y(d x \otimes d s+d s \otimes d x) .
\end{gathered}
$$

Then the set $(\breve{\Psi}, \breve{\xi}, \breve{\eta}, \breve{g})$ is a spacelike almost paracontact structure with index $(\breve{g})=3$ on $R^{5}$. Suppose $N$ is a hypersurface of $R^{5}$ defined by $y=t$. The radical distribution RadTN is spanned by

$$
E=\frac{\partial}{\partial y}+\frac{\partial}{\partial t}
$$

The lightlike transversal vector bundle $\operatorname{ltr} T N$ is spanned by 


$$
\zeta=\frac{1}{2}\left(-\frac{\partial}{\partial y}+\frac{\partial}{\partial t}\right)
$$

and the screen bundle $S(T N)$ is spanned by

$$
\left\{U_{1}, U_{2}, \breve{\xi}\right\}
$$

where $U_{1}=\partial / \partial x$ and $U_{2}=\partial / \partial z$. Also,

$$
\breve{\Psi} E=-E, \quad \breve{\Psi} \zeta=-\zeta
$$

Consequently, $N$ becomes an invariant lightlike hypersurface.

Now, we obtain a necessary and sufficient condition for a lightlike hypersurface to be invariant.

Theorem 8. A hypersurface $N$ of an indefinite almost paracontact metric manifold $(\breve{N}, \breve{\Psi}, \breve{\xi}, \breve{\eta}, \breve{g}, \varepsilon)$ is invariant if and only if

$$
\breve{\Psi} \operatorname{RadTN}=\operatorname{RadTN} \quad \text { and } \quad \breve{\Psi} \operatorname{ltr} T N=\operatorname{ltr} T N .
$$

Proof. Suppose $N$ is an invariant lightlike hypersurface of $\breve{N}$. From (42), for any $X \in \Gamma(T N)$, we get $g(f \breve{\Psi} E, f X)=0$ (that is, there is no component of $\breve{\Psi} E$ in $S(T N)$ ) and $\breve{\Psi} \operatorname{RadTN}=\operatorname{RadTN}$. We write

$$
\breve{\Psi} N=f \breve{\Psi} N+\breve{g}(\breve{\Psi} \zeta, \zeta) E+\breve{g}(\breve{\Psi} \zeta, E) \zeta
$$

where $\zeta$ is a local section of $\operatorname{ltrTN}$. From (50), for any $X \in \Gamma(T N)$, we get $g(f \breve{\Psi} N, f X)=0$, that is, there is no component of $\breve{\Psi} N$ in $S(T N)$. Applying $\breve{\Psi}$ to (50), we get

$$
2 \breve{g}(\breve{\Psi} \zeta, \zeta) \breve{g}(\breve{\Psi} \zeta, E)=0
$$

Since $\operatorname{ker} \breve{\Psi}=\operatorname{Span}\{\breve{\xi}\}$, we obtain $\breve{g}(\breve{\Psi} \zeta, \zeta)=0$. Thus we get $\breve{\Psi} \zeta=\breve{g}(\breve{\Psi} \zeta, E) \zeta$, that is, $\breve{\Psi} \operatorname{ltr} T N=\operatorname{ltr} T N$.

Conversely, let $\breve{\Psi} \operatorname{RadTN}=\operatorname{RadTN}$ and $\breve{\Psi} \operatorname{ltr} T N=\operatorname{ltr} T N$. Then for every $X \in$ $\Gamma(S(T N))$ we get 


$$
\breve{g}(\breve{\Psi} X, E)=\breve{g}(X, \breve{\Psi} E)=0 ;
$$

thus there is no component of $\breve{\Psi} X$ in $\operatorname{ltr} T N$. Similarly, one obtains

$$
\breve{g}(\breve{\Psi} X, \zeta)=\breve{g}(X, \breve{\Psi} \zeta)=0,
$$

which implies that there is no component of $\breve{\Psi} X$ in RadTN. This completes the proof.

Theorem 9. Let $(\breve{N}, \breve{\Psi}, \breve{\xi}, \breve{\eta}, \breve{g}, \varepsilon)$ be an indefinite almost paracontact metric manifold and $N$ be an invariant lightlike hypersurface of $\breve{N}$. Then $(N, \phi, \breve{\xi}, \breve{\eta}, g, \varepsilon)$ is an indefinite almost paracontact metric manifold.

Proof. Suppose that $N$ is a lightlike hypersurface of $\breve{N}$, which is invariant. In view of (38), it follows that

$$
\breve{\Psi} X=\phi X, \quad X \in \Gamma(T N) .
$$

Using (18) and (51), we have

$$
\phi^{2} X=X-\breve{\eta}(X) \breve{\xi}
$$

Also from (51), it follows that

$$
\phi \breve{\xi}=0
$$

Next, in view of (52) and (53) one can easily see that

$$
\begin{gathered}
\breve{\eta} \circ \phi=0, \\
\breve{\eta}(\breve{\xi})=1 .
\end{gathered}
$$

Moreover, from (44) we have

$$
g(\phi X, \phi Y)=g(X, Y)-\varepsilon \breve{\eta}(X) \breve{\eta}(Y)
$$

for all $X, Y \in \Gamma(T N)$. This completes the proof.

Proposition 10. Let $N$ be an invariant lightlike hypersurface of an indefinite para Sasakian manifold $(\breve{N}, \breve{\Psi}, \breve{\xi}, \breve{\eta}, \breve{g}, \varepsilon)$. Then

$$
g\left(A_{\zeta} f X, \breve{\xi}\right)=0, \quad X \in \Gamma(T N)
$$


Proof. Because of $g(\breve{\xi}, \zeta)=0$, using (27), we get

$$
\breve{g}\left(\breve{\nabla}_{X} \zeta, \breve{\xi}\right)=-\varepsilon \breve{g}(\zeta, \breve{\Psi} X)
$$

Now from (7), we give the conclusion as follows.

Theorem 11. An invariant lightlike hypersurface of an indefinite para Sasakian manifold is always indefinite para Sasakian. Also,

$$
\begin{gathered}
B(X, \phi Y) \zeta-B(X, Y) \breve{\Psi} \zeta=0, \\
\phi\left(A_{\zeta} X\right)=A_{\breve{\Psi} \zeta} X-w(X) \breve{\xi}
\end{gathered}
$$

for all $X, Y \in \Gamma(T N)$.

Proof. We have

$$
\begin{aligned}
\left(\breve{\nabla}_{X} \breve{\Psi}\right) Y & =\breve{\nabla}_{X} \breve{\Psi} Y-\breve{\Psi}\left(\breve{\nabla}_{X} Y\right) \\
& =\breve{\nabla}_{X} \breve{\Psi} Y-\breve{\Psi}\left(\nabla_{X} Y+B(X, Y) \zeta\right) \\
& =\nabla_{X} \phi Y+B(X, \phi Y) \zeta-\phi \nabla_{X} Y-B(X, Y) \breve{\Psi} \zeta \\
& =\left(\nabla_{X} \phi\right) Y+B(X, \phi Y) \zeta-B(X, Y) \breve{\Psi} \zeta .
\end{aligned}
$$

Using (26) in the above equation one gets

$$
-g(\phi X, \phi Y) \breve{\xi}-\varepsilon \breve{\eta}(Y) \phi^{2} X=\left(\nabla_{X} \phi\right) Y+B(X, \phi Y) \zeta-B(X, Y) \breve{\Psi} \zeta
$$

When we equate tangential parts in (56), we get

$$
\left(\nabla_{X} \phi\right) Y=-g(\phi X, \phi Y) \breve{\xi}-\varepsilon \breve{\eta}(Y) \phi^{2} X
$$

In view of (57) and Theorem 9, we see that $N$ is indefinite para Sasakian. When we equate transversal parts in (56), we get (54).

Taking into account (26) and (7), one gets

$$
\begin{aligned}
-w(X) \breve{\xi} & =\left(\breve{\nabla}_{X} \breve{\Psi}\right) \zeta=\breve{\nabla}_{X} \breve{\Psi} \zeta-\breve{\Psi}\left(\breve{\nabla}_{X} \zeta\right) \\
& =-A_{\breve{\Psi} \zeta} X+\breve{g}\left(\breve{\nabla}_{X} \breve{\Psi} \zeta, E\right) \zeta+\breve{\Psi}\left(A_{\zeta} X\right)-v(X) \breve{\Psi} \zeta .
\end{aligned}
$$


After equating the tangential parts, one obtains

$$
-w(X) \breve{\xi}=-A_{\breve{\Psi} \zeta} X+\phi\left(A_{\zeta} X\right)
$$

which cocludes the proof.

Remark 12. It is well-understood that, if there is a lightlike hypersurface in an indefinite Sasakian manifold, then the dimension of the Sasakian manifold must be greater than or equal to 5. However, for the existence of lightlike hypersurfaces of an indefinite paracontact metric manifold, such an obstruction on the dimension of the ambient manifold disappears.

\section{Screen Semi-İnvariant Lightlike Hypersurfaces}

We begin with the following.

Definition 13. Let $(\breve{N}, \breve{\Psi}, \breve{\xi}, \breve{\eta}, \breve{g}, \varepsilon)$ be an $(n+2)$-dimensional indefinite almost paracontact metric manifold and $N$ be a lightlike hypersurface of $\breve{N}$. If $\breve{\Psi} R a d T N \subset$ $S(T N)$ and $\breve{\Psi} \operatorname{ltr} T N \subset S(T N)$, then $N$ is called a screen semi-invariant lightlike hypersurface of $\widetilde{N}$.

Example 14. Let $\mathrm{R}^{5}$ be the 5-dimensional real number space with a coordinate system $(x, y, z, t, s)$. We define

$$
\begin{gathered}
\breve{\eta}=\frac{1}{2}(z d x+t d y+d s), \quad \breve{\xi}=2 \frac{\partial}{\partial s} \\
\breve{\Psi} X=-X_{3} \frac{\partial}{\partial x}-X_{4} \frac{\partial}{\partial y}-X_{1} \frac{\partial}{\partial z}-X_{2} \frac{\partial}{\partial t}+\left(X_{3} z+X_{4} t\right) \frac{\partial}{\partial s} \\
\breve{g}=-\frac{1}{4}(d x \otimes d x+d z \otimes d z)+\frac{1}{4}(d y \otimes d y+d t \otimes d t)+\breve{\eta} \otimes \breve{\eta} .
\end{gathered}
$$

Here $X$ is a vector field given by

$$
X=X_{1} \frac{\partial}{\partial x}+X_{2} \frac{\partial}{\partial y}+X_{3} \frac{\partial}{\partial z}+X_{4} \frac{\partial}{\partial t}+X_{5} \frac{\partial}{\partial s}
$$

Then $(\breve{\Psi}, \breve{\xi}, \breve{\eta}, \breve{g})$ is a spacelike almost paracontact structure on $R^{5}$ and $\operatorname{index}(\breve{g})=2$. 
Now consider a hypersurface $N$ given by

$$
t=z
$$

Then the tangent bundle $T N$ of $N$ is spanned by

$$
\left\{U_{1}=\frac{\partial}{\partial x}, U_{2}=\frac{\partial}{\partial y}, U_{3}=\frac{\partial}{\partial z}+\frac{\partial}{\partial t}, U_{4}=\frac{\partial}{\partial s}\right\},
$$

and $\operatorname{RadTN}$ is spanned by $E=U_{3}$. Here, the lightlike transversal vector bundle is given by

$$
\operatorname{ltrTN}=\operatorname{Span}\left\{\zeta=2\left(-\frac{\partial}{\partial z}+\frac{\partial}{\partial t}\right)\right\}
$$

Furthermore

$$
\begin{aligned}
& \breve{\Psi} E=-\left(\frac{\partial}{\partial x}+\frac{\partial}{\partial y}\right)+(z+t) \frac{\partial}{\partial s} \in \Gamma(S(T N)), \\
& \breve{\Psi} \zeta=2\left(\frac{\partial}{\partial x}-\frac{\partial}{\partial y}+(t-z) \frac{\partial}{\partial s}\right) \in \Gamma(S(T N)) .
\end{aligned}
$$

Thus $M$ is a screen semi-invariant lightlike hypersurface of $R^{5}$.

Let $N$ be a screen semi-invariant lightlike hypersurface of an $(n+2)$-dimensional indefinite almost paracontact metric manifold $\widetilde{N}$. We set

$$
V=\breve{\Psi} E \quad \text { and } \quad U=\breve{\Psi} \zeta
$$

Then, from the second equation of (4) and (22), we obtain

$$
g(V, U)=1
$$

Therefore $\langle V\rangle \oplus\langle U\rangle$ is a non-degenerate vector subbundle of $S(T N)$ of rank 2 . Since $\breve{\xi}$ belongs to $S(T N)$ and

$$
g(V, \breve{\xi})=g(U, \breve{\xi})=0
$$

then there exists a non-degenerate distribution $D_{0}$ of rank $n-3$ on $N$ such that 


$$
S(T N)=D_{0} \perp\{\langle V\rangle \oplus\langle U\rangle\} \perp\langle\breve{\xi}\rangle .
$$

We note that $D_{0}$ is an invariant distribution with respect to $\breve{\Psi}$, that is, $\breve{\Psi} D_{0}=D_{0}$. Denoting

$$
D=D_{0} \perp \operatorname{RadTN} \perp\langle V\rangle \quad \text { and } \quad D^{\prime}=\langle U\rangle,
$$

we have

$$
T N=D \oplus D^{\prime} \perp\langle\breve{\xi}\rangle .
$$

Thus, using projections $R: T N \rightarrow D$ and $Q: T N \rightarrow D^{\prime}$, every $X \in \Gamma(T N)$ can be expressed as

$$
X=R X+Q X+\breve{\eta}(X) \breve{\xi}
$$

yielding

$$
\phi X=\breve{\Psi} R X, \quad X \in \Gamma(T N) .
$$

From (19), (38) and (39), we obtain

$$
\breve{\Psi}^{2} X=\phi^{2} X+u(X) U+\mu(\phi X) \zeta
$$

Equalize tangential and transversal parts in (62) one obtains

$$
\begin{gathered}
\phi^{2}=I-\breve{\eta} \otimes \breve{\xi}-\mu \otimes U, \\
\mu \circ \phi=0,
\end{gathered}
$$

respectively. Afterwards, from (20) one can easily see that

$$
\phi \breve{\xi}=0 \text { and } \mu(\breve{\xi})=0
$$

Since $\breve{\Psi}^{2} \zeta=\zeta$, by using (38) we also have

$$
\phi U=0 \text { and } \mu(U)=1 .
$$

Further, in view of (21), it follows that

$$
\breve{\eta}(U)=0 .
$$


Finally, one obtains

$$
(\breve{\eta} \circ \phi) X=\breve{\eta}(\breve{\Psi} X-\mu(X) \zeta),
$$

yielding

$$
\breve{\eta} \circ \phi=0 .
$$

Now, these conclusions can be narrated as the following

Proposition 15. Let $N$ be a screen semi-invariant lightlike hypersurface of an indefinite almost paracontact metric manifold $(\breve{N}, \breve{\Psi}, \breve{\xi}, \breve{\eta}, \breve{g}, \varepsilon)$. Then $M$ is equipped with a para $(\phi, \breve{\xi}, \breve{\eta}, U, \mu)$-structure, that is,

$$
\begin{array}{cl}
\phi^{2}=I-\breve{\eta} \otimes \breve{\xi}-\mu \otimes U, \quad \phi \breve{\xi}=0, \quad \phi U=0, & \breve{\eta} \circ \phi=0, \\
\mu \circ \phi=0, & \breve{\eta}(\breve{\xi})=1, \quad \mu(U)=1, \quad \breve{\eta}(U)=0, \quad \mu(\breve{\xi})=0 .
\end{array}
$$

Next, we present the following Theorem:

Theorem 16. If $(\breve{N}, \breve{\Psi}, \breve{\xi}, \breve{\eta}, \breve{g}, \varepsilon)$ is an indefinite para Sasakian manifold and $N$ is a screen semi-invariant lightlike hypersurface of it, then

$$
\begin{aligned}
&\left(\nabla_{X} \phi\right) Y= \mu(Y) A_{\zeta} X+B(X, Y) U \\
&-g(X, Y) \breve{\xi}+2 \varepsilon \breve{\eta}(X) \breve{\eta}(Y) \breve{\xi}-\varepsilon \breve{\eta}(Y) X, \\
&\left(\nabla_{X} \phi\right) Y= \mu(Y) A_{\zeta} X+B(X, Y) U \\
&-(g(\phi X, \phi Y)+\mu(X) w(\phi Y)+\mu(Y) w(\phi X)) \breve{\xi} \\
&- \varepsilon \breve{\eta}(Y)\left(\phi^{2} X+\mu(X) U\right), \\
&\left(\nabla_{X} \mu\right) Y=-B(X, \phi Y)-\mu(Y) v(X), \\
& \nabla_{X} U=-\phi\left(A_{\zeta} X\right)+v(X) U, \\
& B(X, U)=-\mu\left(A_{\zeta} X\right),
\end{aligned}
$$

for all $X, Y \in \Gamma(T N)$. 
Proof. We have

$$
\begin{aligned}
\left(\widetilde{\nabla}_{X} \breve{\Psi}\right) Y= & \left(\nabla_{X} \phi\right) Y-\mu(Y) A_{N} X-B(X, Y) U \\
& +\left\{\left(\nabla_{X} \mu\right) Y+\mu(Y) v(X)+B(X, \phi Y)\right\} \zeta,
\end{aligned}
$$

where (38), (6), (7) and (58) are used. Next, from (26) we have

$$
\left(\breve{\nabla}_{X} \breve{\Psi}\right) Y=-g(X, Y) \breve{\xi}+2 \varepsilon \breve{\eta}(X) \breve{\eta}(Y) \breve{\xi}-\varepsilon \breve{\eta}(Y) X, \quad X, Y \in \Gamma(T N)
$$

Using (45), (62) and (64) in (26), for all $X, Y \in \Gamma(T N)$, we also have

$$
\begin{aligned}
\left(\breve{\nabla}_{X} \breve{\Psi}\right) Y= & -(g(\phi X, \phi Y)+\mu(X) w(\phi Y)+\mu(Y) w(\phi X)) \breve{\xi} \\
& -\varepsilon \breve{\eta}(Y)\left(\phi^{2} X+\mu(X) U\right) .
\end{aligned}
$$

From (74) and (75) we get (69). Similarly, from (74) and (76) we get (70). Next, taking transversal part in (74) to be zero, we get (71).

Using (6), (7) and (38) we get

$$
\begin{aligned}
\left(\widetilde{\nabla}_{X} \breve{\Psi}\right) \zeta= & \nabla_{X} U+\phi\left(A_{\zeta} X\right)-v(X) U \\
& +\left(B(X, U)+\mu\left(A_{\zeta} X\right)\right) \zeta
\end{aligned}
$$

Since, from (26) we have

$$
\left(\breve{\nabla}_{X} \breve{\Psi}\right) \zeta=0
$$

then using (77) and (78) we have

$$
0=\nabla_{X} U+\phi\left(A_{\zeta} X\right)-v(X) U+\left(B(X, U)+\mu\left(A_{\zeta} X\right)\right) \zeta
$$

By equating tangential and transversal parts in the above equation we obtain (72) and (73), respectively.

Proposition 17. For a screen semi-invariant lightlike hypersurface $N$ of an indefinite para Sasakian metric manifold $(\breve{N}, \breve{\Psi}, \breve{\xi}, \breve{\eta}, \breve{g}, \varepsilon)$, the Lie derivative of $g$ with respect to the vector field $V$ satisfies

$$
\left(L_{V} g\right)(X, Y)=X(\mu(Y))+Y(\mu(X))+\mu([X, Y])-2 \mu\left(\nabla_{X} Y\right),
$$


for all $X, Y \in \Gamma(T N)$.

Proof. We have

$$
\left(L_{Z} \breve{g}\right)(X, Y)=\left(L_{Z} g\right)(X, Y), \quad X, Y, Z \in \Gamma(T N)
$$

Hence, using $V=\breve{\Psi} E$ and $\breve{g}\left(\left(\widetilde{\nabla}_{X} \breve{\Psi}\right) E, Y\right)=0$ we have

$$
\left(L_{V} g\right)(X, Y)=\left(L_{V} \breve{g}\right)(X, Y)=\breve{g}\left(\breve{\nabla}_{X} E, \breve{\Psi} Y\right)+\breve{g}\left(\breve{\Psi} X, \breve{\nabla}_{Y} E\right)
$$

Next,

$$
\begin{aligned}
\breve{g}\left(\breve{\nabla}_{X} E, \breve{\Psi} Y\right) & =\breve{g}\left(\breve{\nabla}_{X} E, \phi Y+\mu(Y) \zeta\right) \\
& =\breve{g}\left(\breve{\nabla}_{X} E, \phi Y\right)+\mu(Y) \breve{g}\left(\breve{\nabla}_{X} E, \zeta\right) \\
& =-\breve{g}\left(E, \breve{\nabla}_{X} \phi Y\right)-\mu(Y) \breve{g}\left(E, \breve{\nabla}_{X} \zeta\right) \\
& =-B(X, \phi Y)-\mu(Y) v(X) \\
& =\left(\nabla_{X} \mu\right) Y,
\end{aligned}
$$

which gives

$$
\breve{g}\left(\breve{\nabla}_{X} E, \breve{\Psi} Y\right)=X(\mu(Y))-\mu\left(\nabla_{X} Y\right)
$$

Using (82) in (81) we complete the proof.

\subsection{Integrability of $D \perp\langle\breve{\xi}\rangle$}

We note that $X \in \Gamma(D \perp\langle\breve{\xi}\rangle)$ if and only if $\mu(X)=0$. Now from (71), we have

$$
\mu\left(\nabla_{X} Y\right)=\nabla_{X} \mu(Y)+B(X, \phi Y)+\mu(Y) v(X)
$$

for all $X, Y \in \Gamma(T M)$. From the above equation we get

$$
\begin{aligned}
\mu[X, Y]= & B(X, \phi Y)-B(\phi X, Y) \\
& +\nabla_{X} \mu(Y)-\nabla_{Y} \mu(X)+\mu(Y) v(X)-\mu(X) v(Y)
\end{aligned}
$$

Now, let $X, Y \in \Gamma(D \perp\langle\breve{\xi}\rangle)$. Then $\mu(X)=0=\mu(Y)$, and from the previous equation we get 


$$
\mu[X, Y]=B(X, \phi Y)-B(\phi X, Y)
$$

for all $X, Y \in \Gamma(D \perp\langle\breve{\xi}\rangle)$. Thus we get a necessary and sufficient condition for the integrability of the distribution $D \perp\langle\breve{\xi}\rangle$ in the following:

Theorem 18. For a screen semi-invariant lightlike hypersurface $N$ of an indefinite para Sasakian manifold $(N, \breve{\Psi}, \breve{\xi}, \breve{\eta}, \breve{g}, \varepsilon)$, the distribution $D \perp\langle\breve{\xi}\rangle$ is integrable if and only if

$$
B(X, \phi Y)=B(\phi X, Y), \quad X, Y \in \Gamma(D \perp\langle\breve{\xi}\rangle)
$$

\subsection{Integrability of $D^{\prime} \perp\langle\breve{\xi}\rangle$}

Here we find a necessary and sufficient condition for the distribution $D^{\prime} \perp\langle\breve{\xi}\rangle$ to be integrable.

Theorem 19. For a screen semi-invariant lightlike hypersurface $N$ of an indefinite para Sasakian manifold $(\breve{N}, \breve{\Psi}, \breve{\xi}, \breve{\eta}, \breve{g}, \varepsilon)$, the distribution $D^{\prime} \perp\langle\breve{\xi}\rangle$ is integrable if and only if

$$
A_{\zeta} \breve{\xi}+\varepsilon U=0
$$

Proof. Note that $X \in \Gamma\left(D^{\prime} \perp\langle\breve{\xi}\rangle\right)$ if and only if $\phi X=0$. Now for all $X, Y \in \Gamma(T M)$, in view of (69), we have

$$
\begin{aligned}
\phi\left(\nabla_{X} Y\right)= & \nabla_{X} \phi Y-\mu(Y) A_{\zeta} X-B(X, Y) U \\
& +g(X, Y) \breve{\xi}-2 \varepsilon \breve{\eta}(X) \breve{\eta}(Y) \breve{\xi}+\varepsilon \breve{\eta}(Y) X .
\end{aligned}
$$

From the above equation we get

$$
\phi[X, Y]=\nabla_{X} \phi Y-\nabla_{Y} \phi X+\mu(X) A_{\zeta} Y-\mu(Y) A_{\zeta} X+\varepsilon \breve{\eta}(Y) X-\varepsilon \breve{\eta}(X) Y .
$$

In particular, for $X, Y \in \Gamma\left(D^{\prime} \perp\langle\breve{\xi}\rangle\right)$ we get

$$
\phi[X, Y]=\mu(X) A_{\zeta} Y-\mu(Y) A_{\zeta} X+\varepsilon \breve{\eta}(Y) X-\varepsilon \breve{\eta}(X) Y .
$$

But $D^{\prime}$ and $\langle\breve{\xi}\rangle$ are integrable, hence $D^{\prime} \perp\langle\breve{\xi}\rangle$ is integrable if and only if 


$$
\phi[U, \breve{\xi}]=0
$$

which, in view of (84), is equivalent to (83).

\section{Acknowledgement}

The third author (MMT) is thankful to TUBITAK for financial support partly, as this study was carried out during the visit of the third author (July 13-28, 2009) to İnönü University, Malatya, Turkey.

\section{References}

[1] Küpeli, D.N., On null submanifolds in spacetimes, Geometriae Dedicata, 23(1), 33-51, 1987.

[2] Bejancu, A., Duggal, K.L., Real hypersurfaces of indefinite Kaehler manifolds, International Journal of Mathematics and Mathematical Sciences, 16(3), 545-556, 1993.

[3] Duggal, K.L., Şahin, B., Differential geometry of lightlike submanifolds, Frontiers in Mathematics. Birkhäuser Verlag, Basel, 2010.

[4] Sasaki, S., On differentiable manifolds with certain structures which are closely related to almost contact structure I, Tohoku Mathematical Journal, (2) 12, 459-476, 1960.

[5] Blair, D.E., Riemannian geometry of contact and symplectic manifolds (Second edition) Progress in Mathematics, 203. Birkhauser Boston, Inc., Boston, MA, 2010.

[6] Satō, I., On a structure similar to the almost contact structure, Tensor (N.S.), 30(3), 219-224, 1976.

[7] Takahashi, T., Sasakian manifold with pseudo-Riemannian metric, Tohoku Mathematical Journal, 21(2), 271-290, 1969.

[8] Duggal, K.L., Bejancu, A., Lightlike submanifolds of semi-Riemannian manifolds and its applications, Mathematics and its Applications, 364. Kluwer Academic Publishers Group, Dordrecht, 1996.

[9] Duggal, K.L., Space time manifolds and contact structures, International Journal of Mathematics and Mathematical Sciences, 13(3), 545-553, 1990.

[10] Duggal, K.L., Şahin, B., Lightlike submanifolds of indefinite Sasakian manifolds, International Journal of Mathematics and Mathematical Sciences, Art. ID 57585, 21 pp., 2007. 
[11] Kang, T.H., Jung, S.D., Kim, B.H., Pak, H.K., Pak, J.S., Lightlike hypersurfaces of indefinite Sasakian manifolds, Indian Journal of Pure and Applied Mathematics, 34 (9), 1369-1380, 2003.

[12] Matsumoto, K., On Lorentzian paracontact manifolds, Bullet in of the Yamagata University, Natural Science, 12(2), 151-156, 1989.

[13] Tripathi, M.M., Kılıç, E., Yüksel Perktaş, S., Keleş, S., Indefinite almost paracontact metric manifolds, International Journal of Mathematics and Mathematical Sciences, Art. Id. 846195, pp. 19, 2010.

[14] Yüksel Perktaş, S., Kılıç, E., Tripathi, M.M., Keleş, S., On indefinite - para Sasakian 3 -manifolds, International Journal of Pure and Applied Mathematics, 77(4), 485-499, 2012.

[15] Shukla, S.S., Yadev, A., Radical transversal lightlike submanifolds of indefinite para-Sasakian manifolds, Demonstratio Mathematica, vol. XVLVII, No. 4, 994-1011, 2014.

[16] Shukla, S.S., Yadev, A., Lightlike submanifolds of indefinite para-Sasakian manifolds, Matematicki Vesnik, 66 (4), 371-386, 2014.

[17] O’Neill, B., Semi-Riemannian geometry with applications to relativity, Pure and Applied Mathematics, 103. Academic Press, Inc. [Harcourt Brace Jovanovich, Publishers], New York, 1983.

[18] Duggal, K.L., Jin, D.H., Null Curves and Hypersurfaces of Semi-Riemannian Manifolds, World Scientific Publishing Co. Pte. Ltd., Hackensack, NJ, 2007.

[19] Beem, J.K., Ehrlich, P.E., Global Lorentzian geometry, Monographs and Textbooks in Pure and Applied Mathematics, 67. Marcel Dekker, New York, 1981. 